\title{
Bayesian estimation for the burr type III distribution under type II Doubly censored data
}

\author{
Reza Azimi ${ }^{1 *}$, Farhad Yaghmaei ${ }^{2}$ \\ ${ }^{1}$ Department of Statistics, Parsabad Moghan Branch, Islamic Azad University, Parsabad Moghan, Iran \\ ${ }^{2}$ Faculty of science, Golestan University, Gorgan, Golestan, Iran \\ *Corresponding author E-mail: Azimireza1365@gmail.com
}

\begin{abstract}
In this paper, we consider the problem of estimating the parameters and reliability function of the burr Type III distribution based on Type II Doubly censored sample. We use the Bayesian procedures to obtain the estimators of parameter and reliability function of burr Type III distribution under symmetric (squared-error) and asymmetric (Linex and general entropy)loss functions.
\end{abstract}

Keywords: Burr Type III Distribution, Type II Doubly censored, Bayesian estimation, Asymmetric loss function.

\section{Introduction}

The cumulative distribution function(CDF), probability density function (PDF) and reliability function of The Burr Type III distribution are given, respectively, by (see Burr [4] or lindsay et al., [5])

$$
\begin{aligned}
& F(x ; \theta, c)=\left(1+x^{-c}\right)^{-\theta}, \quad x>0, \theta>0, c>0 \\
& f(x ; \theta, c)=\theta c x^{-(c+1)}\left(1+x^{-c}\right)^{-(\theta+1)} \\
& R(t ; \theta, c)=1-\left(1+t^{-c}\right)^{-\theta} .
\end{aligned}
$$

Consider a random sample of size $n$ from Burr Type III distribution and let $x_{r}, \ldots, x_{s}$ be the ordered observations remaining when the $(r-1)$ smallest observations and the $(n-s)$ largest observations have been censored. The likelihood function of $\theta$ for type II doubly censored sample $x=\left(x_{r}, x_{r+1}, \ldots, x_{s}\right)$ is given by (see Fernandez [2] or kim and song [3] )

$$
L(\theta \mid \mathbf{X})=\frac{n !}{(r-1) !(n-s) !}\left[F\left(x_{r}\right)\right]^{r-1}\left[1-F\left(x_{s}\right)\right]^{n-s} \prod_{i=r}^{s} f\left(x_{i}\right) .
$$

\section{Likelihood, prior and posterior}

Substituting (1) and (2) into (4), the likelihood function becomes to be proportional to

$$
L(\theta \mid \mathbf{X}) \propto \theta^{s-r+1} e^{-\theta\left(r \ln \left(1+x_{r}^{-c}\right)+\sum_{i=r}^{s} \ln \left(1+x_{i}^{-c}\right)\right)}\left[1-\left(1+x_{s}^{-c}\right)^{-\theta}\right]^{n-s}
$$

It is assumed that the parameter $\theta$ has a gamma prior distribution with the shape and scale parameters as $a$ and $b$, respectively and it has the PDF

$$
\pi(\theta)=\frac{b^{a}}{\Gamma(a)} \theta^{a-1} e^{-b \theta}
$$


Combining (5) and (6) the posterior density function of $\theta$ takes the form

$$
\pi(\theta \mid x)=\frac{(b+w)^{s^{\prime}+a}}{\Gamma\left(s^{\prime}+a\right) \psi_{V}\left(w, s^{\prime}+a\right)} \theta^{s^{\prime}+a-1} e^{-\theta(b+w)}\left[1-e^{-\theta V\left(x_{s}\right)}\right]^{n-s}
$$

where,

$$
s^{\prime}=s-r+1, w=r \ln \left(1+x_{r}^{-c}\right)+\sum_{i=r}^{s} \ln \left(1+x_{i}^{-c}\right), V\left(x_{s}\right)=\ln 1+x_{s}^{-c}
$$

and

$$
\psi_{V}\left(w, s^{\prime}+a\right)=(b+w)^{s^{\prime}+a} \sum_{j=0}^{n-s} \frac{(-1)^{j}\left(\begin{array}{c}
n-s \\
j
\end{array}\right)}{\left(b+w+j V\left(x_{s}\right)\right)^{s^{\prime}+a}}, \quad \psi_{0}=1 .
$$

\section{Bayes estimator under squared-error loss function}

Under squared-error loss, the Bayes estimator of $\theta$ is the mean of the posterior density given by

$$
\hat{\theta}_{S}=\frac{s^{\prime}+a}{b+w} \times \frac{\psi_{V}\left(w, s^{\prime}+a+1\right)}{\psi_{V}\left(w, s^{\prime}+a\right)} .
$$

Other problems of interest are those of estimating the reliability function $R(t)$, with fixed $t>0$. under squared-error loss function, the Bayesian estimators of $R(t)$ are found to be

$$
\hat{R}_{S}=1-\left[\left(\frac{b+w}{b+w+V(t)}\right)^{s^{\prime}+a+1} \frac{\psi_{V}\left(w+V(t), s^{\prime}+a\right)}{\psi_{V}\left(w, s^{\prime}+a\right)}\right] .
$$

where $V(t)=\ln 1+t^{-c}$.

\section{Bayes estimator under LINEX loss function}

The LINEX loss function for $\theta$ can be expressed as the following proportional

$$
L(\Delta) \propto \exp (c \Delta)-c \Delta-1 ; \quad c \neq 0
$$

where $\Delta=(\hat{\theta}-\theta)$ and $\hat{\theta}$ is an estimate of $\theta$. The Bayes estimator of $\theta$, denoted by $\hat{\theta_{L}}$ under the LINEX loss function is given by

$$
\hat{\theta_{L}}=-\frac{1}{k} \ln E[\exp (-k \theta)]
$$

For more details about LINEX loss function see Zellner [6]. Under LINEX loss function, we obtain Bayesian estimator of the parameter $\theta$, and reliability function $R(t)$, as the following forms

$$
\begin{gathered}
\hat{\theta}_{L}=-\frac{1}{k} \ln \left[\left(\frac{b+w}{b+w+k}\right)^{s^{\prime}+a} \frac{\psi_{V}\left(w+k, s^{\prime}+a\right)}{\psi_{V}\left(w, s^{\prime}+a\right)}\right] \\
\hat{R}_{l}=1+\frac{1}{k} \ln \left[\sum_{l=0}^{\infty} \frac{(-1)^{l} k^{l}}{l !}\left(\frac{b+w}{b+w+l V(t)}\right)^{s^{\prime}+a} \frac{\psi_{V}\left(w+l V(t), s^{\prime}+a\right)}{\psi_{V}\left(w, s^{\prime}+a\right)}\right]
\end{gathered}
$$

\subsection{Bayes estimator under general entropy loss function (GELF)}

In many practical situations, it appears to be more realistic to express the loss in terms of the ratio $\frac{d}{\theta}$. In this case, Calabria and Pulcini [1], point out that a useful asymmetric loss function is the general entropy loss function,

$$
L(\delta)=\left[\delta^{q}-q \ln (\delta)-1\right], \delta=\frac{d}{\theta} .
$$


The magnitude of $q$ reflects the degree of asymmetry. The Bayes estimator of $\theta$ under the GELF is given by

$$
\hat{\theta}_{G}=\left[E\left(\theta^{-q} \mid \mathbf{x}\right)\right]^{-\frac{1}{q}}
$$

Therefore the Bayesian estimators of parameter $\theta$ and and reliability function $R(t)$ under general entropy loss function are given by

$$
\hat{\theta}_{G}=\left[\frac{\Gamma\left(s^{\prime}+a-q\right)}{\Gamma\left(s^{\prime}+a\right)} \frac{\psi_{V}\left(w, s^{\prime}+a-q\right)}{\psi_{V}\left(w, s^{\prime}+a\right)}\right]^{-\frac{1}{q}} \frac{1}{b+w}
$$

and

$$
\hat{R}_{G}=1-\left[\left(\frac{b+w}{b+w-q V(t)}\right)^{-\frac{s^{\prime}+a}{q}}\left(\frac{\psi_{V}\left(w-q V(t), s^{\prime}+a\right)}{\psi_{V}\left(w, s^{\prime}+a\right)}\right)^{-\frac{1}{q}}\right]
$$

\section{$5 \quad$ Numerical example}

In this section we consider the following simulated type II doubly censored sample of size $n=15(r=4, s=13)$ from the Burr type III distribution with parameters $\theta=1.5$ and $c=2$.

$$
\begin{gathered}
-,-,-, 0.8321534,0.8510093,0.8861234,1.0076263,1.1443972,1.2214424, \\
1.2455378,1.5926474,3.4799892,5.2624189,-,-
\end{gathered}
$$

The first three failure times and the last two are censored. We used the above sample to compute the Bayesian estimators of parameter $\theta$ and reliability function $R(t)$. In all above cases the prior parameters chosen as $a=2$ and $b=1$. The true values of $R(t)$ in $t=0.5$ is obtained $R(0.5)=0.9105573$ The results obtained are given in Table 1 .

Table 1: Bayesian Estimators of parameter $\theta$ and reliability function $R(t)$.

\begin{tabular}{ccc|ccc}
\hline$\hat{\theta}_{S}$ & $\hat{\theta}_{L}(c=-1)$ & $\hat{\theta}_{G}(q=1)$ & $\hat{R}_{G}$ & $\hat{R}_{G}(c=-1)$ & $\hat{R}_{G}(q=1)$ \\
\hline 1.531 & 1.490 & 1.312 & 0.8785 & 0.8853 & 0.9165 \\
\hline
\end{tabular}

\section{Conclusions}

In this paper, We use the Bayesian Estimation method under different loss functions to obtain the estimators of the parameter and reliability function of Burr type III distribution based on Type II Doubly censored samples. A numerical example in section 5 to illustrate our approach.

\section{References}

[1] R. Calabria, G. Pulcini, Point estimation under asymmetric loss functions for left-truncated exponential samples, Communications in Statistics Theory and Methods 25, 585-600, 1996.

[2] A. J. Fernandez, Bayesian inference from type II censored Rayleigh data. Statistics and Probability Letters, 48, 393-399, 2000.

[3] C. kim and S. song, Bayesian estimation of the parameters of the generalized exponential distribution from doubly censored samples, Stat Papers DOI 10.1007/s00362-008-0142-3.

[4] I. W. Burr, Cumulative frequency distribution, Annals of Mathematical Statistics, 13, pp. 215-232, 1942.

[5] S. R. Lindsay, G. R. Wood and R. C. Woollons, modeling the diameter distribution of forest stands using the burr distribution, journal of applied statistics, 23, 609-619, 1996.

[6] A. Zellner, Bayes estimation and prediction using asymmetric loss functions, Journal of the American Statistical Association, 81, 446-451, 1986. 